\title{
Quasi-Bi-Hamiltonian Structures of the 2-Dimensional Kepler Problem ${ }^{\star}$
}

\author{
Jose F. CARIÑENA and Manuel F. RAÑADA \\ Departamento de Física Teórica and IUMA, Universidad de Zaragoza, 50009 Zaragoza, Spain \\ E-mail: jfc@unizar.es,mfran@unizar.es
}

Received September 29, 2015, in final form January 25, 2016; Published online January 27, 2016

http://dx.doi.org/10.3842/SIGMA.2016.010

\begin{abstract}
The existence of quasi-bi-Hamiltonian structures for the Kepler problem is studied. We first relate the superintegrability of the system with the existence of two complex functions endowed with very interesting Poisson bracket properties and then we prove the existence of a quasi-bi-Hamiltonian structure by making use of these two functions. The paper can be considered as divided in two parts. In the first part a quasi-bi-Hamiltonian structure is obtained by making use of polar coordinates and in the second part a new quasi-bi-Hamiltonian structure is obtained by making use of the separability of the system in parabolic coordinates.
\end{abstract}

Key words: Kepler problem; superintegrability; complex structures; bi-Hamiltonian structures; quasi-bi-Hamiltonian structures

2010 Mathematics Subject Classification: 37J15; 37J35; 70H06; 70H33

\section{Introduction}

\subsection{A geometric introduction}

In differential geometric terms, the phase space $M$ of a Hamiltonian system is the $2 n$-dimensional cotangent bundle $M=T^{*} Q$ of the $n$-dimensional configuration space $Q$. Cotangent bundles are manifolds endowed, in a natural or canonical way, with a symplectic structure $\omega_{0}$; if $\left\{\left(q_{i}\right) \mid i=\right.$ $2, \ldots, n\}$ are local coordinates in $Q$ and $\left\{\left(q_{j}, p_{j}\right) ; j=1,2, \ldots, n\right\}$ the induced coordinates in $T^{*} Q$, then $\omega_{0}$ is given by

$$
\omega_{0}=d q_{j} \wedge d p_{j}, \quad \omega_{0}=-d \theta_{0}, \quad \theta_{0}=p_{j} d q_{j},
$$

(we write all the indices as subscripts and summation convention on the repeated index is used). Given a differentiable function $F$ in $T^{*} Q, F=F(q, p)$, the vector field $X_{F}$ uniquely defined as the solution of the equation

$$
i\left(X_{F}\right) \omega_{0}=d F
$$

is called the Hamiltonian vector field of the function $F$. In particular, given a Hamiltonian $H=H(q, p)$, the dynamics is given by the corresponding Hamiltonian vector field $X_{H}$, that is, $i\left(X_{H}\right) \omega_{0}=d H$.

A vector field $\Gamma \in \mathfrak{X}\left(T^{*} Q\right)$ is Hamiltonian if there is a function $H$ such that $\Gamma=X_{H}$, i.e., $i(\Gamma) \omega_{0}=d H$, and locally-Hamiltonian when $i(\Gamma) \omega_{0}$ is a closed 1-form. This is equivalent to $\Gamma$-invariance of $\omega_{0}$, i.e., $\mathcal{L}_{\Gamma} \omega_{0}=0$.

A system of differential equations is called bi-Hamiltonian if it can be written in two different ways in Hamiltonian form. Suppose a manifold $M$ equipped with two different symplectic

\footnotetext{
${ }^{\star}$ This paper is a contribution to the Special Issue on Analytical Mechanics and Differential Geometry in honour of Sergio Benenti. The full collection is available at http://www.emis.de/journals/SIGMA/Benenti.html
} 
structures $\omega_{0}$ and $\omega_{1}$. A vector field $\Gamma$ on $T^{*} Q$ is said to be a bi-Hamiltonian vector field if it is Hamiltonian with respect to both symplectic structures, i.e.,

$$
i(\Gamma) \omega_{0}=d H_{0} \quad \text { and } \quad i(\Gamma) \omega_{1}=d H_{1} .
$$

The two functions, $H_{0}$ and $H_{1}$, are integrals of motion for $\Gamma$. A weaker form of bi-Hamiltonian system is when the only symplectic form is the first one ( $\omega_{1}$ is a closed but nonsymplectic 2 -form).

We point out that an important example of bi-Hamiltonian system is the rational harmonic oscillator (non-central harmonic oscillator with rational ratio of frequencies) [8]

$$
H=\frac{1}{2}\left(p_{x}^{2}+p_{y}^{2}\right)+\frac{1}{2} \alpha_{0}^{2}\left(m^{2} x^{2}+n^{2} y^{2}\right) .
$$

A symplectic form determines a Poisson bivector $\Lambda$ that satisfies the vanishing of the Schouten bracket $[\Lambda, \Lambda]=0$ (this property is equivalent to the Jacobi identity); we note that there exist non-constant rank Poisson structure not related with symplectic structures. The compatibility condition between two different Poisson structures, $\Lambda_{0}$ and $\Lambda_{1}$, means that the linear combination $\Lambda_{\lambda}=\Lambda_{0}-\lambda \Lambda_{1}$ is a Poisson pencil, that is, it is a Poisson bivector for every value of $\lambda$; therefore the corresponding bracket $\{\cdot, \cdot\}_{\lambda}=\{\cdot, \cdot\}_{0}-\lambda\{\cdot, \cdot\}_{1}$ is a pencil of Poisson brackets.

Bi-Hamiltonian systems are systems endowed with very interesting properties but, in general, it is quite difficult to find a bi-Hamiltonian formulation for a given Hamiltonian vector field, and for this reason it is useful to introduce the concept of quasi-bi-Hamiltonian system.

Definition 1. A vector field $X$ on a symplectic manifold $(M, \omega)$ is called quasi-Hamiltonian if there exists a (nowhere-vanishing) function $\mu$ such that $\mu X$ is a Hamiltonian vector field

$$
\mu X \in \mathfrak{X}_{H}(M) .
$$

Thus $i(\mu X) \omega=d h$ for some function $h$.

We call $\mu$ an integrating factor of the quasi-Hamiltonian system, because it is an integrating factor for the 1-form $i(X) \omega$, and we note that in this case the function $h$ is a first integral of $X$. Note that this condition can alternatively be written as as $i(X)(\mu \omega)=d h$, but the point is that the 2 -form $\mu \omega$ is not closed in the general case.

The scarcity of bi-Hamiltonian systems leads to relax the condition for the vector field being Hamiltonian to a simpler situation of quasi-Hamiltonian with respect to the second symplectic structure.

Definition 2. A Hamiltonian vector field $X$ on a symplectic manifold $(M, \omega)$ is called quasi-biHamiltonian if there exist another symplectic structure $\omega_{1}$, and a nowhere-vanishing function $\mu$, such that $\mu X$ is a Hamiltonian vector field with respect to $\omega_{1}$.

This concept was first introduced in [4] in the particular case of systems with two degrees of freedom and it was quickly extended in $[23,24]$ for a higher-dimensional systems. Some recent papers considering properties of this particular class of systems are $[1,2,3,4,5,6,8,14,15,23$, $24,25,36]$.

The nondegeneracy of the canonical form $\omega_{0}$ provides a vector bundle isomorphism $\widehat{\omega_{0}}$ of $T\left(T^{*} Q\right)$ on $T^{*}\left(T^{*} Q\right)$, inducing an identification of vector fields and 1-forms on the phase space. A consequence is that the pair $\left(\omega_{0}, \omega_{1}\right)$ determines a $(1,1)$ tensor field $R$ defined as $R={\widehat{\omega_{0}}}^{-1} \circ \widehat{\omega}_{1}$, that is,

$$
\omega_{1}(X, Y)=\omega_{0}(R X, Y), \quad \forall X, Y \in \mathfrak{X}\left(T^{*} Q\right) .
$$


Note that in the definition of $R$ the 2 -form $\omega_{1}$ is necessarily closed but it can be nonsymplectic. If $\Gamma$ is bi-Hamiltonian with respect to $\left(\omega_{0}, \omega_{1}\right)$ then $R$ is $\Gamma$-invariant, that is, $\mathcal{L}_{\Gamma} R=0$, where $\mathcal{L}$ denotes the Lie derivative (this means that the characteristic polynomial of $R$ is an invariant for $\Gamma$, and consequently the coefficients of the polynomial are constants of motion). The Nijenhuis tensor $N_{R}$ of the tensor field $R$ is defined by

$$
N_{R}(X, Y)=R^{2}[X, Y]+[R X, R Y]-R[R X, Y]-R[X, R Y]
$$

It has been proved that if $\Gamma$ is a Hamiltonian dynamical system of the type described above and such that (i) The tensor $N_{R}$ of $R$ vanishes, (ii) The tensor field $R$ has $n$ distinct eigenfunctions (that is, they are maximally distinct), then the eigenfunctions of $R$ are in involution and the system is therefore completely integrable $[12,16,17,18,21]$. It is important to note that the eigenvalues of $R$ are constants of motion for $\Gamma$ even in the case that the two properties (i) and (ii) are not satisfied (but then the eigenfunctions are not in involution).

It is known that the Liouville formalism characterize the Hamiltonians that are integrable but it does not provide a method for obtaining the integrals of motion; therefore it has been necessary to elaborate different methods for obtaining constants of motion (Hamilton-Jacobi separability, Lax pairs formalism, Noether symmetries, Hidden symmetries, etc); the existence of a bi-Hamiltonian structure with the above two mentioned properties (Nijenhuis torsion condition and maximally distinct eigenvalues) can be considered as method to establish the Liouville integrability of a system; because of this, these two properties are frequently included in the definition of a bi-Hamiltonian structure.

Most of systems admitting a bi-Hamiltonian structure are separable systems; so these two properties (separability and double Hamiltonian structure) are properties very close related (see [18] for a detailed discussion of this question and [35] for the case of multiple separability). Quasi-bi-Hamiltonian systems are very less known than the bi-Hamiltonian ones but it seems that they are also related with separability. Nevertheless, in this case the tensor field $R$ is not $\Gamma$-invariant and the eigenvalues of the tensor field $R$ are not constants of motion. An interesting property is that it was shown in [4] that in the particular case of two degrees of freedom the function $\mu^{2} / \operatorname{det} R$ is a constant of the motion.

The potential of the Kepler problem is spherically symmetric and therefore it admits alternative Lagrangians (the existence of alternative Lagrangians for central potentials is studied in $[13,20,26])$; recall that if there exist alternative Lagrangian descriptions then one can find non-Noether constants of motion [7]. This system has been studied as a bi-Hamilltonian system by making use of different approaches; Rauch-Wojciechowski proved the existence of a bi-Hamiltonian formulation but introducing an extra variable so that the phase space is odddimensional and the Poisson brackets are degenerate [34] and more recently [19] a bi-Hamiltonian formulation for the perturbed Kepler problem has also been studied by making use of Delaunaytype variables.

\subsection{Structure and purpose of the paper}

Now in this paper we will analyze certain properties of the Kepler problem related with the existence of quasi-bi-Hamiltonian structures. The following two points summarize the contents of the paper.

- First, we will study the existence of certain complex functions with interesting Poisson properties and then we will prove that the superintegrability of the system is directly related with the properties of these complex functions. As is well-known this system is multi-separable (it separates in both polar and parabolic coordinates); so we first present the study making use of polar coordinates and then we undertake a similar study by 
employing parabolic coordinates (the parabolic complex functions are different from the polar ones).

- Second, we prove that the above mentioned complex functions determine the existence of several quasi-bi-Hamiltonian structures. This is done in two steps: first with complex 2 -forms and then with several real 2-forms. The properties of these geometric structures and of the associated recursion operators are analyzed.

It is important to note that this study is concerned with the existence of quasi-bi-Hamiltonian structures (instead of bi-Hamiltonian). So we recall that if $i(\Gamma) \omega_{1}=\lambda d H_{1}$ then $\mathcal{X}_{\Gamma} \omega_{1}=d \lambda \wedge d H_{1} \neq 0$. Consequently the tensor field $R$ is not $\Gamma$-invariant and the eigenvalues of $R$ are not constants of motion.

We must clearly say that the structures obtained by this method (wedge product of the differentials of complex functions) do not satisfy the above mentioned Nijenhuis torsion condition. So perhaps it is convenient to name them as weak quasi-bi-Hamiltonian structures (in opposition to strong structures satisfying the Nijenhuis condition). Nevertheless, the purpose in this paper is not to prove the integrability of a system as consequence of a bi-Hamiltonian structure, since it is perfectly known that the Kepler problem is not only integrable but also superintegrable. The purpose is to study new and interesting properties of the Kepler problem. In fact, it has been proved that if a dynamical vector field satisfies certain properties (existence of canonoid transformations [9, 10] or existence of non-symplectic symmetries [27, 28]) then it is Hamiltonian with respect to two different structures without satisfying necessarily the Nijenhuis condition.

The structure of the paper is as follows: In Section 2 we study the Kepler problem by making use of polar coordinates $(r, \phi)$; we relate the superintegrability of the system with the existence of two complex functions $M_{r}$ and $N_{\phi}$ endowed with very interesting Poisson bracket properties and then we prove the existence of a quasi-bi-Hamiltonian structure making use of these two functions. Then in Section 3 we consider once more the same system but in terms of parabolic coordinates $(a, b)$ and we obtain a new quasi-bi-Hamiltonian structure (different to the previous one) making use of a similar technique but with new complex functions $M_{a}$ and $M_{b}$. Finally in Section 4 we make some final comments.

\section{From superintegrability to quasi-bi-Hamiltonian structures}

After these rather general comments we restrict our study to the Kepler problem in the Euclidean plane that, as it is well known, is superintegrable and multiseparable (polar and parabolic coordinates).

Let us first notice that in some cases the two-dimensional Euclidean systems possess certain interesting properties. For example, if the potential $V(x, y)$ takes the form $V=A(u)+B(v)$, $u=x+y, v=x-y$, then it admits a new Hamiltonian structure (and also a Lax pair) [22]; unfortunately the new structure is in most of cases constant (we mean that the new Poisson bracket is determined by a symplectic form as $\omega_{1}=d x \wedge d p_{y}+d y \wedge d p_{x}$ ).

In the next paragraphs we will relate the existence of bi-Hamiltonian structures with the properties of the two complex functions with interesting Poisson bracket properties.

\subsection{Complex functions and superintegrabilty}

It is well known that the Hamiltonian of the two-dimensional Kepler problem

$$
H_{K}=\frac{1}{2}\left(p_{r}^{2}+\frac{p_{\phi}^{2}}{r^{2}}\right)+V_{K}, \quad V_{K}=-\frac{g}{r}, \quad 0<g \in \mathbb{R},
$$


is Hamilton-Jacobi (H-J) separable in polar coordinates $(r, \phi)$ and it is, therefore, Liouville integrable with the angular momentum $J_{2}=p_{\phi}$ as the associate constant of motion. Moreover, it is also known that it is a super-integrable system with the two components of the LaplaceRunge-Lenz vector as additional integrals of motion. Now we will prove that this property of superintegrability can be related to the existence of certain complex functions with interesting Poisson bracket properties.

Let us denote by $M_{r j}$ and $N_{\phi j}, j=1,2$, the following real functions

$$
M_{r 1}=p_{r} p_{\phi}, \quad M_{r 2}=g-\frac{p_{\phi}^{2}}{r}, \quad \text { and } \quad N_{\phi 1}=\cos \phi, \quad N_{\phi 2}=\sin \phi
$$

Then we have the following properties

$$
\begin{aligned}
& \text { (i) } \frac{d}{d t} M_{r 1}=\left\{M_{r 1}, H_{K}\right\}=-\lambda M_{r 2}, \quad \frac{d}{d t} M_{r 2}=\left\{M_{r 2}, H_{K}\right\}=\lambda M_{r 1} \text {, } \\
& \text { (ii) } \frac{d}{d t} N_{\phi 1}=\left\{N_{\phi 1}, H_{K}\right\}=-\lambda N_{\phi 2}, \quad \frac{d}{d t} N_{\phi 2}=\left\{N_{\phi 2}, H_{K}\right\}=\lambda N_{\phi 1} \text {, }
\end{aligned}
$$

where $\lambda$ denote the following function

$$
\lambda=\frac{p_{\phi}}{r^{2}} .
$$

The property (ii), representing the behaviour of the angular functions $N_{\phi j}$, is true for all the central potentials $V(r)$; but the property (i), behaviour of the functions $M_{r j}$, is specific of the potential of the Kepler problem.

Consider next the complexification of the linear space of functions on the manifold and extend by bilinearity the Poisson bracket. If we denote $M_{r}$ and $N_{\phi}$ the complex functions

$$
M_{r}=M_{r 1}+i M_{r 2}, \quad N_{\phi}=N_{\phi 1}+i N_{\phi 2},
$$

then they have the following properties

$$
\left\{M_{r}, H_{K}\right\}=i \lambda M_{r}, \quad\left\{N_{\phi}, H_{K}\right\}=i \lambda N_{\phi},
$$

and consequently the Poisson bracket of $M_{r} N_{\phi}^{*}$ with the Kepler Hamiltonian vanishes

$$
\left\{M_{r} N_{\phi}^{*}, H_{K}\right\}=\left\{M_{r}, H_{K}\right\} N_{\phi}^{*}+M_{r}\left\{N_{\phi}^{*}, H_{K}\right\}=\left(i \lambda M_{r}\right) N_{\phi}^{*}+M_{r}\left(-i \lambda N_{\phi}^{*}\right)=0 .
$$

We can summarize this result in the following proposition.

Proposition 1. Let us consider the Hamiltonian of the Kepler problem

$$
H_{K}=\frac{1}{2}\left(p_{r}^{2}+\frac{p_{\phi}^{2}}{r^{2}}\right)+V_{K}, \quad V_{K}=-\frac{g}{r},
$$

Then, the complex function $J_{34}$ defined as

$$
J_{34}=M_{r} N_{\phi}^{*}
$$

is a (complex) constant of the motion.

Of course $J_{34}$ determines two real first-integrals

$$
J_{34}=J_{3}+i J_{4}, \quad\left\{J_{3}, H_{K}\right\}=0, \quad\left\{J_{4}, H_{K}\right\}=0,
$$


whose coordinate expressions turn out to be

$$
\begin{aligned}
& J_{3}=\operatorname{Re}\left(J_{34}\right)=p_{r} p_{\phi} \cos \phi-\frac{p_{\phi}^{2}}{r} \sin \phi+g \sin \phi, \\
& J_{4}=\operatorname{Im}\left(J_{34}\right)=p_{r} p_{\phi} \sin \phi+\frac{p_{\phi}^{2}}{r} \cos \phi-g \cos \phi .
\end{aligned}
$$

That is, the two functions $J_{3}$ and $J_{4}$ are just the two components of the two-dimensional LaplaceRunge-Lenz vector.

Summarizing, we have got two interesting properties. First, the superintegrability of the Kepler problem is directly related with the existence of two complex functions whose Poisson brackets with the Hamiltonian are proportional with a common complex factor to themselves, and second, the two components of the Laplace-Runge-Lenz vector appear as the real and imaginary parts of the complex first-integral of motion. Remark that $N_{\phi}$ is a complex function of constant modulus one, while the modulus of $M_{r}$ is a polynomial of degree four in the momenta given by

$$
M_{r} M_{r}^{*}=\left(p_{r} p_{\phi}\right)^{2}+\left(g-\frac{p_{\phi}^{2}}{r}\right)^{2}=2 p_{\phi}^{2} H_{K}+g^{2} .
$$

\subsection{Complex functions and quasi-bi-Hamiltonian structures}

Let us denote by $Y_{34}$ the (complex) Hamiltonian vector field of $J_{34}$

$$
i\left(Y_{34}\right) \omega_{0}=d J_{34}
$$

that obviously satisfies $Y_{34}\left(H_{K}\right)=\left\{H_{K}, J_{34}\right\}=0$, and by $Y_{r}$ and $Y_{\phi}$ the Hamiltonian vector fields of $M_{r}$ and $N_{\phi}$ :

$$
i\left(Y_{r}\right) \omega_{0}=d M_{r}, \quad i\left(Y_{\phi}\right) \omega_{0}=d N_{\phi}
$$

Their local coordinate expressions are, respectively, given by

$$
Y_{r}=p_{\phi} \frac{\partial}{\partial r}+\left(p_{r}-2 i \frac{p_{\phi}}{r}\right) \frac{\partial}{\partial \phi}-i\left(\frac{p_{\phi}^{2}}{r^{2}}\right) \frac{\partial}{\partial p_{r}},
$$

and

$$
Y_{\phi}=(\sin \phi-i \cos \phi) \frac{\partial}{\partial p_{\phi}} .
$$

Then, the vector field $Y_{34}$ appears as a linear combination of $Y_{r}$ and $Y_{\phi}^{*}$; more specifically we have

$$
Y_{34}=N_{\phi}^{*} Y_{r}+M_{r} Y_{\phi}^{*}=Y+Y^{\prime}, \quad Y=N_{\phi}^{*} Y_{r}, \quad Y^{\prime}=M_{r} Y_{\phi}^{*} .
$$

The vector field $Y_{34}$ is certainly a symmetry of the Hamiltonian system $\left(T^{*} Q, \omega_{0}, H_{K}\right)$, but the two vector fields, $Y$ and $Y^{\prime}$, are neither symmetries of the symplectic form $\omega_{0}$ (that is, $\mathcal{X}_{Y} \omega_{0} \neq 0$ and $\mathcal{X}_{Y^{\prime}} \omega_{0} \neq 0$ ) nor symmetries of the Hamiltonian (that is, $\mathcal{X}_{Y} H_{K} \neq 0$ and $\mathcal{X}_{Y^{\prime}} H_{K} \neq 0$ ). Moreover, remark that they are not symmetries of the dynamics, because

$$
\left[Y, \Gamma_{K}\right] \neq 0, \quad\left[Y^{\prime}, \Gamma_{K}\right] \neq 0, \quad i\left(\Gamma_{K}\right) \omega_{0}=d H_{K} .
$$

More specifically: 
Proposition 2. The Lie bracket of the dynamical vector field $\Gamma_{K}$ with $Y$ is given by

$\left[\Gamma_{K}, Y\right]=i J_{34} X_{\lambda}$,

where $X_{\lambda}$ is the Hamiltonian vector field of the function $\lambda$.

Proof. A direct computation leads to

$$
\left[\Gamma_{K}, Y\right]=\Gamma_{K}\left(N_{\phi}^{*}\right) Y_{r}+N_{\phi}^{*}\left[\Gamma_{K}, Y_{r}\right]=-i \lambda N_{\phi}^{*} Y_{r}+N_{\phi}^{*}\left(-X_{\left\{H_{K}, M_{r}\right\}}\right) .
$$

where we have used that the Lie bracket of two Hamiltonian vector fields satisfies $\left[X_{f}, X_{g}\right]=$ $-X_{\{f, g\}}$. Note also that the Hamiltonian vector field of a product $f g$ is given by $X_{f g}=$ $f X_{g}+g X_{f}$, and then the above Lie bracket becomes

$$
\left[\Gamma_{K}, Y\right]=-i \lambda N_{\phi}^{*} Y_{r}+i N_{\phi}^{*}\left(X_{\lambda M_{r}}\right)=-i \lambda N_{\phi}^{*} Y_{r}+i N_{\phi}^{*}\left(\lambda Y_{r}+M_{r} X_{\lambda}\right)=i\left(M_{r} N_{\phi}^{*}\right) X_{\lambda} .
$$

The vector field $X_{\lambda}$ on the right hand side represents an obstruction for $Y$ to be a dynamical symmetry. Only when $\lambda$ be a numerical constant the vector field $Y$ (and also $Y^{\prime}$ ) is a dynamical symmetry of $\Gamma_{K}$.

In the following $\Omega$ will denote the complex 2 -form defined as

$$
\Omega=d M_{r} \wedge d N_{\phi}^{*}
$$

The two complex 2-forms $\omega_{Y}$ and $\omega_{Y}^{\prime}$ obtained by Lie derivative of $\omega_{0}$, i.e.,

$$
\mathcal{L}_{Y} \omega_{0}=\omega_{Y}, \quad \mathcal{L}_{Y^{\prime}} \omega_{0}=\omega_{Y}^{\prime},
$$

are such

$$
\begin{aligned}
& \mathcal{L}_{Y} \omega_{0}=i_{Y}\left(d \omega_{0}\right)+d\left(i_{Y} \omega_{0}\right)=d\left(i_{Y} \omega_{0}\right)=d\left(N_{\phi}^{*} d M_{r}\right)=-\Omega, \\
& \mathcal{L}_{Y^{\prime}} \omega_{0}=i_{Y^{\prime}}\left(d \omega_{0}\right)+d\left(i_{Y^{\prime}} \omega_{0}\right)=d\left(i_{Y^{\prime}} \omega_{0}\right)=d\left(M_{r} d N_{\phi}^{*}\right)=\Omega .
\end{aligned}
$$

Using the preceding results we can prove:

Proposition 3. The Hamiltonian vector field $\Gamma_{K}$ of the Kepler problem is a quasi-Hamiltonian system with respect to the complex 2 -form $\Omega$.

Proof. The contraction of the vector field $\Gamma_{K}$ with the complex 2-form $\Omega$ gives

$$
i\left(\Gamma_{K}\right) \Omega=\Gamma_{K}\left(M_{r}\right) d N_{\phi}^{*}-\Gamma_{K}\left(N_{\phi}^{*}\right) d M_{r},
$$

and recalling that

$$
\Gamma_{K}\left(M_{r}\right)=\left\{M_{r}, H_{K}\right\}=i \lambda M_{r}, \quad \Gamma_{K}\left(N_{\phi}^{*}\right)=\left\{N_{\phi}^{*}, H_{K}\right\}=-i \lambda N_{\phi}^{*},
$$

we arrive to

$$
i\left(\Gamma_{K}\right) \Omega=\left(i \lambda M_{r}\right) d N_{\phi}^{*}+\left(i \lambda N_{\phi}^{*}\right) d M_{r}=i \lambda d\left(M_{r} N_{\phi}^{*}\right) .
$$

The complex 2 -form $\Omega$ can be written as

$$
\Omega=\Omega_{1}+i \Omega_{2}
$$

where the two real 2-forms, $\Omega_{1}=\operatorname{Re}(\Omega)$ and $\Omega_{2}=\operatorname{Im}(\Omega)$, take the form

$$
\Omega_{1}=d M_{r 1} \wedge d N_{\phi 1}+d M_{r 2} \wedge d N_{\phi 2}=d\left(p_{r} p_{\phi}\right) \wedge d(\cos \phi)+d\left(g-\frac{p_{\phi}^{2}}{r}\right) \wedge d(\sin \phi)
$$




$$
\begin{aligned}
& =\alpha_{12} d r \wedge d \phi+\alpha_{23} d \phi \wedge d p_{r}+\alpha_{24} d \phi \wedge d p_{\phi}, \\
\Omega_{2} & =-d M_{r 1} \wedge d N_{\phi 2}+d M_{r 2} \wedge d N_{\phi 1}=-d\left(p_{r} p_{\phi}\right) \wedge d(\sin \phi)+d\left(g-\frac{p_{\phi}^{2}}{r}\right) \wedge d(\cos \phi) \\
& =\beta_{12} d r \wedge d \phi+\beta_{23} d \phi \wedge d p_{r}+\beta_{24} d \phi \wedge d p_{\phi}
\end{aligned}
$$

with $\alpha_{i j}$ and $\beta_{i j}$ being given by

$$
\alpha_{12}=\left(\frac{p_{\phi}^{2}}{r^{2}}\right) \cos \phi, \quad \alpha_{23}=p_{\phi} \sin \phi, \quad \alpha_{24}=p_{r} \sin \phi+2\left(\frac{p_{\phi}}{r}\right) \cos \phi,
$$

and

$$
\beta_{12}=-\left(\frac{p_{\phi}^{2}}{r^{2}}\right) \sin \phi, \quad \beta_{23}=p_{\phi} \cos \phi, \quad \beta_{24}=p_{r} \cos \phi-2\left(\frac{p_{\phi}}{r}\right) \sin \phi .
$$

Then we have

$$
i\left(\Gamma_{K}\right) \Omega_{1}=-\lambda d J_{4}, \quad i\left(\Gamma_{K}\right) \Omega_{2}=\lambda d J_{3},
$$

what means that $\Gamma_{K}$ is also quasi-bi-Hamiltonian with respect to the two real 2 -forms $\left(\omega_{0}, \Omega_{1}\right)$ or $\left(\omega_{0}, \Omega_{2}\right)$.

Remark that the complex 2-form $\Omega$ is well defined but it is not symplectic. In fact, from the above expressions in coordinates we have $\Omega_{1} \wedge \Omega_{1}=0, \Omega_{2} \wedge \Omega_{2}=0$, and $\Omega_{1} \wedge \Omega_{2}=0$, and therefore we obtain

$$
\Omega \wedge \Omega=\left(\Omega_{1} \wedge \Omega_{1}-\Omega_{2} \wedge \Omega_{2}\right)+2 i \Omega_{1} \wedge \Omega_{2}=0 .
$$

The distribution defined by the kernel of $\Omega$, that is two-dimensional, is given by

$$
\operatorname{Ker} \Omega=\left\{f_{1} Z_{1}+f_{2} Z_{2} \mid f_{1}, f_{2}: \mathbb{R}^{2} \times \mathbb{R}^{2} \rightarrow \mathbb{C}\right\},
$$

where the vector fields $Z_{1}$ and $Z_{2}$ are

$$
Z_{1}=\left(\alpha_{23}+i \beta_{23}\right) \frac{\partial}{\partial r}+\left(\alpha_{12}+i \beta_{12}\right) \frac{\partial}{\partial p_{r}}, \quad Z_{2}=\left(\alpha_{24}+i \beta_{24}\right) \frac{\partial}{\partial r}+\left(\alpha_{12}+i \beta_{12}\right) \frac{\partial}{\partial p_{\phi}} .
$$

Therefore it satisfies

$\left[\operatorname{Ker} \Omega, \Gamma_{K}\right] \subset \operatorname{Ker} \Omega$.

That is, $\Gamma_{K}$ preserves the distribution $\operatorname{Ker} \Omega$.

If $Y_{3}$ and $Y_{4}$ are the Hamiltonian vector fields (with respect to the canonical symplectic form $\omega_{0}$ ) of the first integrals $J_{3}$ and $J_{4}$, then the dynamical vector field $\Gamma_{K}$ is orthogonal to $Y_{4}$ with respect to the structure $\Omega_{1}$ and it is also orthogonal to $Y_{3}$ with respect to the structure $\Omega_{2}$, that is,

$$
i\left(\Gamma_{K}\right) i\left(Y_{4}\right) \Omega_{1}=0, \quad i\left(\Gamma_{K}\right) i\left(Y_{3}\right) \Omega_{2}=0 .
$$

Just to close the section we remark that had we applied this technique to the isotropic twodimensional harmonic oscillator with frequency $\alpha$ we had obtained the function $M_{r}$ as

$$
M_{r}=\left(\frac{2}{r} p_{r} p_{\phi}\right)+i\left(p_{r}^{2}-\frac{p_{\phi}^{2}}{r^{2}}+\alpha^{2} r^{2}\right),
$$

(the angular function $N_{\phi}$ would be the same) and the constants so obtained are but the components of the Fradkin tensor. This shows that the harmonic oscillator is an example of dynamical system both bi-Hamiltonian and quasi-bi-Hamiltonian. 


\subsection{Recursion operators and some comments}

The bi-Hamiltonian structure $\left(\omega_{0}, \Omega\right)$ determines a complex recursion operator $R$ defined as

$$
\Omega(X, Y)=\omega_{0}(R X, Y), \quad \forall X, Y \in \mathfrak{X}\left(T^{*} Q\right) .
$$

But as $\Omega$ and $R$ are complex, we can introduce two real recursion operator $R_{1}$ and $R_{2}$ defined as

$$
\Omega_{1}(X, Y)=\omega_{0}\left(R_{1} X, Y\right), \quad \Omega_{2}(X, Y)=\omega_{0}\left(R_{2} X, Y\right) .
$$

We recall that $\widehat{\omega_{0}}$ is the map $\widehat{\omega_{0}}: \mathfrak{X}\left(T^{*} Q\right) \rightarrow \wedge^{1}\left(T^{*} Q\right)$ given by contraction, that is $\widehat{\omega_{0}}(X)=$ $i(X) \omega_{0}$, and then the nondegenerate character of $\omega_{0}$ means that the map $\widehat{\omega_{0}}$ is a bijection. Using this notation we can write the two operators $R_{1}$ and $R_{2}$ as follows

$$
R_{1}={\widehat{\omega_{0}}}^{-1} \circ \widehat{\Omega}_{1}, \quad R_{2}={\widehat{\omega_{0}}}^{-1} \circ \widehat{\Omega}_{2}
$$

Then we have the following properties

(i) The coordinates expressions of $R_{1}$ and $R_{2}$ are

$$
\begin{aligned}
R_{1}= & -\alpha_{12} \frac{\partial}{\partial p_{\phi}} \otimes d r+\left[\alpha_{23} \frac{\partial}{\partial r}+\alpha_{24} \frac{\partial}{\partial \phi}+\alpha_{12} \frac{\partial}{\partial p_{r}}\right] \otimes d \phi \\
& +\alpha_{23} \frac{\partial}{\partial p_{\phi}} \otimes d p_{r}+\alpha_{24} \frac{\partial}{\partial p_{\phi}} \otimes d p_{\phi}
\end{aligned}
$$

and

$$
\begin{aligned}
R_{2}= & -\beta_{12} \frac{\partial}{\partial p_{\phi}} \otimes d r+\left[\beta_{23} \frac{\partial}{\partial r}+\beta_{24} \frac{\partial}{\partial \phi}+\beta_{12} \frac{\partial}{\partial p_{r}}\right] \otimes d \phi \\
& +\beta_{23} \frac{\partial}{\partial p_{\phi}} \otimes d p_{r}+\beta_{24} \frac{\partial}{\partial p_{\phi}} \otimes d p_{\phi} .
\end{aligned}
$$

(ii) $R_{1}$ and $R_{2}$ have two different eigenvalues doubly degenerate and one of them is null (that is, $\left.\lambda_{1}=\lambda_{2}=0, \lambda_{3}=\lambda_{4} \neq 0\right)$. Therefore we have

$$
\operatorname{det}\left[R_{1}\right]=\operatorname{det}\left[R_{2}\right]=0,
$$

what is a consequence of the singular character of $\Omega_{1}$ and $\Omega_{2}$.

We close this section summarizing the situation we have arrived. We have first introduced two complex functions, $M_{r}$ and $N_{\phi}$, mainly because of the behaviour of their Poisson brackets. Then we have proved that they are interesting for two reasons: first because they determine the existence of superintegrability (existence of additional constants of motion) and second because they determine quasi-bi-Hamiltonian structures (first complex $\left(\omega_{0}, \Omega\right)$ and then real $\left.\left(\omega_{0}, \Omega_{1}, \Omega_{2}\right)\right)$.

Concerning the first point, in this case the additional constants of motion are just the components of the Runge-Lenz vector (that have been highly studied making use of different approaches). Now we have arrived to a new property: they can also be obtained as a consequence of this complex formalism.

Concerning the second point, the two complex functions $M_{r}$ and $N_{\phi}$ determine the above mentioned geometric structures (first complex and then real) but unfortunately they are degenerated (we recall that $\Omega_{1} \wedge \Omega_{1}=0, \Omega_{2} \wedge \Omega_{2}=0$ ). This can be considered as a limitation of 
these geometric structures. If a bi-Hamiltonians structure satisfies all the appropriate properties (that is, symplectic forms, vanishing of the Nijenhuis torsion of the recursion operator $R$, diagonalizable recursion operator $R$ with functionally independent real eigenvalues) then it determines the Liouville integrability of the system. In fact, the aim of the approach presented in this paper is not to prove the integrability of a system as consequence of a bi-Hamiltonian structure as we start with a system known to be not only integrable but also superintegrable. The existence of $\left(\Omega_{1}, \Omega_{2}\right)$ must be considered, not as a method for arriving to the integrability of the system, but as a new and interesting property of the Kepler problem (for the moment only of the two-dimensional system, the generalization to the three-dimensional case must be considered as an open question).

\section{New complex functions and new quasi-bi-Hamiltonian structures}

In this section we will study the existence of new bi-Hamiltonian structures for the Kepler dynamics by making use of parabolic coordinates $(a, b)$ defined as

$$
x=\frac{1}{2}\left(a^{2}-b^{2}\right), \quad y=a b .
$$

Of course all previous results can be translated to this new language in such a way that the functions $M_{r 1}$ and $M_{r 2}$ are now given by

$$
M_{r 1}=\frac{\left(a p_{b}-b p_{a}\right)\left(a p_{a}+b p_{b}\right)}{\sqrt{a^{2}+b^{2}}}, \quad M_{r 2}=\frac{\left(a p_{b}-b p_{a}\right)^{2}}{\sqrt{a^{2}+b^{2}}}-g,
$$

while functions $N_{\phi 1}$ and $N_{\phi 2}$ become

$$
N_{\phi 1}=\frac{a^{2}-b^{2}}{\sqrt{a^{2}+b^{2}}}, \quad N_{\phi 2}=\frac{2 a b}{\sqrt{a^{2}+b^{2}}} .
$$

But the important point is that the behaviour of the Kepler Hamiltonian in these coordinates will permit us to obtain new results different from the previous ones.

\subsection{Complex functions and superintegrability}

The general form of a natural Euclidean Hamiltonian is

$$
H=\frac{1}{2 m}\left(\frac{p_{a}^{2}+p_{b}^{2}}{a^{2}+b^{2}}\right)+V(a, b) .
$$

in such a way that if the potential $V$ is of the form

$$
V(a, b)=\frac{A(a)+B(b)}{a^{2}+b^{2}},
$$

then the Hamiltonian is Hamilton-Jacobi separable and it is, therefore, Liouville integrable with the following quadratic function

$$
J_{2}=\frac{1}{\left(a^{2}+b^{2}\right)}\left(a p_{b}-b p_{a}\right)\left(a p_{b}+b p_{a}\right)+2\left(\frac{a^{2} B-b^{2} A}{a^{2}+b^{2}}\right)
$$

as the second constant of motion (the first one is the Hamiltonian itself). 
For simplifying the following expressions we introduce the following notation:

$$
J=a p_{b}-b p_{a}, \quad P_{x}=\frac{a p_{a}-b p_{b}}{a^{2}+b^{2}}, \quad P_{y}=\frac{a p_{b}+b p_{a}}{a^{2}+b^{2}} .
$$

The Hamiltonian of the Kepler problem when written in parabolic coordinates is

$$
H_{K}=\frac{1}{2}\left(\frac{p_{a}^{2}+p_{b}^{2}}{a^{2}+b^{2}}\right)+V_{K}, \quad V_{K}=-\frac{g}{a^{2}+b^{2}},
$$

and the Kepler dynamics is given by the following vector field

$$
\Gamma_{K}=\left(\frac{p_{a}}{a^{2}+b^{2}}\right) \frac{\partial}{\partial a}+\left(\frac{p_{b}}{a^{2}+b^{2}}\right) \frac{\partial}{\partial b}+\left(\frac{p_{a}^{2}+p_{b}^{2}-2 g}{\left(a^{2}+b^{2}\right)^{2}}\right) a \frac{\partial}{\partial p_{a}}+\left(\frac{p_{a}^{2}+p_{b}^{2}-2 g}{\left(a^{2}+b^{2}\right)^{2}}\right) b \frac{\partial}{\partial p_{b}},
$$

in such a way that, as $\omega_{0}=d a \wedge d p_{a}+d b \wedge d p_{b}$, we have

$$
i\left(\Gamma_{K}\right)\left(d a \wedge d p_{a}+d b \wedge d p_{b}\right)=d H_{K} .
$$

The Hamiltonian $H_{K}$ is Hamilton-Jacobi separable in coordinates $(a, b)$ and the associated quadratic constant of motion is the component $R_{x}$ of the Laplace-Runge-Lenz vector

$$
R_{x}=J P_{y}-g\left(\frac{a^{2}-b^{2}}{a^{2}+b^{2}}\right) .
$$

Let us now introduce the functions $M_{a j}$ and $M_{b j}, j=1,2$, defined by

$$
M_{a 1}=\frac{J p_{a}}{\sqrt{a^{2}+b^{2}}}, \quad M_{a 2}=\frac{2 g a-J p_{b}}{\sqrt{a^{2}+b^{2}}},
$$

and

$$
M_{b 1}=\frac{J p_{b}}{\sqrt{a^{2}+b^{2}}}, \quad M_{b 2}=\frac{2 g b+J p_{a}}{\sqrt{a^{2}+b^{2}}} .
$$

Then, the important property is that the Poisson bracket of the function $M_{a 1}$ with $H_{K}$ is proportional to $M_{a 2}$ while the Poisson bracket of $M_{a 2}$ with $H_{K}$ is proportional to $M_{a 1}$, but with the opposite sign:

$$
\left\{M_{a 1}, H_{K}\right\}=-\lambda M_{a 2}, \quad\left\{M_{a 2}, H_{K}\right\}=\lambda M_{a 1},
$$

and the same is true for the functions $M_{b 1}$ and $M_{b 2}$,

$$
\left\{M_{b 1}, H_{K}\right\}=-\lambda M_{b 2}, \quad\left\{M_{b 2}, H_{K}\right\}=\lambda M_{b 1},
$$

where now $\lambda$ denotes the following function

$$
\lambda=\frac{a p_{b}-b p_{a}}{\left(a^{2}+b^{2}\right)^{2}} .
$$

Therefore the two complex functions $M_{a}$ and $M_{b}$ defined as

$$
M_{a}=M_{a 1}+i M_{a 2}, \quad M_{b}=M_{b 1}+i M_{b 2},
$$

satisfy

$$
\left\{M_{a}, H_{K}\right\}=i \lambda M_{a}, \quad\left\{M_{b}, H_{K}\right\}=i \lambda M_{b} .
$$


Proposition 4. The complex function $K_{34}$ defined as

$$
K_{34}=M_{a} M_{b}^{*}
$$

is a (complex) constant of the motion for the dynamics of the Kepler problem described by the Hamiltonian (1).

We omit the proof because it is quite similar to the proof of the previous Proposition 1.

Note that the modulus of the complex functions $M_{a}$ and $M_{b}$ are given by

$$
M_{a} M_{a}^{*}=2\left(J^{2} H_{K}-g R_{x}+g^{2}\right), \quad M_{b} M_{b}^{*}=2\left(J^{2} H_{K}+g R_{x}+g^{2}\right),
$$

and then

$$
M_{a} M_{a}^{*}+M_{b} M_{b}^{*}=4\left(J^{2} H_{K}+g^{2}\right) .
$$

Of course the complex function $K_{34}$ determines two real functions that are first integrals for the Kepler problem

$$
K_{34}=K_{3}+i K_{4}, \quad\left\{K_{3}, H_{K}\right\}=0, \quad\left\{K_{4}, H_{K}\right\}=0,
$$

with $K_{3}$ and $K_{4}$ given by

$$
\begin{aligned}
& K_{3}=\operatorname{Re}\left(K_{34}\right)=M_{a 1} M_{b 1}+M_{a 2} M_{b 2}=J P_{x}+g\left(\frac{2 a b}{a^{2}+b^{2}}\right), \\
& K_{4}=\operatorname{Im}\left(K_{34}\right)=M_{a 2} M_{b 1}-M_{a 1} M_{b 2}=-2 J^{2} H_{K} .
\end{aligned}
$$

Remark that the function $K_{3}$ is the other Laplace-Runge-Lenz constant, while $K_{4}$, that is a fourth order polynomial in the momenta, determines the angular momentum $J$ as a factor.

\subsection{Complex functions and quasi-bi-Hamiltonian structures}

First we recall that the complex functions $M_{a}$ y $M_{b}$ are given by

$$
M_{a}=\left(\frac{J p_{a}}{\sqrt{a^{2}+b^{2}}}\right)+i\left(\frac{2 g a-J p_{b}}{\sqrt{a^{2}+b^{2}}}\right), \quad M_{b}=\left(\frac{J p_{b}}{\sqrt{a^{2}+b^{2}}}\right)+i\left(\frac{2 g b+J p_{a}}{\sqrt{a^{2}+b^{2}}}\right) .
$$

Let us now denote by $Z_{34}$ the Hamiltonian vector field of the function $K_{34}$, i.e., $i\left(Z_{34}\right) \omega_{0}=$ $d Z_{34}$, such that $Z_{34}\left(H_{K}\right)=0$, and by $Z_{a}$ and $Z_{b}$ the Hamiltonian vector fields of the complex functions $M_{a}$ and $M_{b}$, that is,

$$
i\left(Z_{a}\right) \omega_{0}=d M_{a}, \quad i\left(Z_{b}\right) \omega_{0}=d M_{b}
$$

Their coordinate expressions are given by

$$
Z_{a}=\left(\frac{\partial M_{a}}{\partial p_{a}}\right) \frac{\partial}{\partial a}+\left(\frac{\partial M_{a}}{\partial p_{b}}\right) \frac{\partial}{\partial b}-\left(\frac{\partial M_{a}}{\partial a}\right) \frac{\partial}{\partial p_{a}}-\left(\frac{\partial M_{a}}{\partial b}\right) \frac{\partial}{\partial p_{b}}=Z_{a 1}+i Z_{a 2},
$$

with $Z_{a 1}$ and $Z_{a 2}$ given by

$$
\begin{aligned}
& Z_{a 1}=\frac{1}{\sqrt{a^{2}+b^{2}}}\left(\left(a p_{b}-2 b p_{a}\right) \frac{\partial}{\partial a}+a p_{a} \frac{\partial}{\partial b}-\frac{\left(a p_{a}+b p_{b}\right)}{a^{2}+b^{2}}\left(b p_{a} \frac{\partial}{\partial p_{a}}-a p_{a} \frac{\partial}{\partial p_{b}}\right)\right), \\
& Z_{a 2}=\frac{1}{\sqrt{a^{2}+b^{2}}}\left(b p_{b} \frac{\partial}{\partial a}+\left(b p_{a}-2 a p_{b}\right) \frac{\partial}{\partial b}-\frac{\left(a p_{a}+b p_{b}\right) p_{b}-2 g b}{a^{2}+b^{2}}\left(-b \frac{\partial}{\partial p_{a}}+a \frac{\partial}{\partial p_{b}}\right)\right),
\end{aligned}
$$


and

$$
Z_{b}=\left(\frac{\partial M_{b}}{\partial p_{a}}\right) \frac{\partial}{\partial a}+\left(\frac{\partial M_{b}}{\partial p_{b}}\right) \frac{\partial}{\partial b}-\left(\frac{\partial M_{b}}{\partial a}\right) \frac{\partial}{\partial p_{a}}-\left(\frac{\partial M_{b}}{\partial b}\right) \frac{\partial}{\partial p_{b}}=Z_{b 1}+i Z_{b 2},
$$

with $Z_{b 1}$ and $Z_{b 2}$ given by

$$
\begin{aligned}
& Z_{b 1}=\frac{1}{\sqrt{a^{2}+b^{2}}}\left(-b p_{b} \frac{\partial}{\partial a}+\left(2 a p_{b}-b p_{a}\right) \frac{\partial}{\partial b}-\frac{\left(a p_{a}+b p_{b}\right)}{a^{2}+b^{2}}\left(b p_{b} \frac{\partial}{\partial p_{a}}-a p_{b} \frac{\partial}{\partial p_{b}}\right)\right), \\
& Z_{b 2}=\frac{1}{\sqrt{a^{2}+b^{2}}}\left(\left(a p_{b}-2 b p_{a}\right) \frac{\partial}{\partial a}+a p_{a} \frac{\partial}{\partial b}-\frac{\left(a p_{a}+b p_{b}\right) p_{a}-2 g a}{a^{2}+b^{2}}\left(b \frac{\partial}{\partial p_{a}}-a \frac{\partial}{\partial p_{b}}\right)\right) .
\end{aligned}
$$

Now recalling that

$$
d Z_{34}=d\left(M_{a} M_{b}^{*}\right)=M_{b}^{*} d\left(M_{a}\right)+M_{a} d\left(M_{b}^{*}\right),
$$

we obtain

$$
Z_{34}=M_{b}^{*} Z_{a}+M_{a} Z_{b}^{*}=Z+Z^{\prime}, \quad \text { where } \quad Z=M_{b}^{*} Z_{a}, \quad Z^{\prime}=M_{a} Z_{b}^{*} .
$$

The following proposition is similar to that of Proposition 2 and we omit the proof.

Proposition 5. The Lie bracket of the Kepler dynamical vector field $\Gamma_{K}$ with the vector field $Z$ is given by

$$
\left[\Gamma_{K}, Z\right]=i K_{34} X_{\lambda}
$$

where $X_{\lambda}$ is the Hamiltonian vector field of $\lambda$ solution of the equation $i\left(X_{\lambda}\right) \omega_{0}=d \lambda$.

In the following we will denote by $\Omega_{a b}$ the complex 2 -form defined as

$$
\begin{aligned}
\Omega_{a b} & =d M_{a} \wedge d M_{b}^{*} \\
& =d\left[\left(\frac{J p_{a}}{\sqrt{a^{2}+b^{2}}}\right)+i\left(\frac{2 g a-J p_{b}}{\sqrt{a^{2}+b^{2}}}\right)\right] \wedge d\left[\left(\frac{J p_{b}}{\sqrt{a^{2}+b^{2}}}\right)-i\left(\frac{2 g b+J p_{a}}{\sqrt{a^{2}+b^{2}}}\right)\right] .
\end{aligned}
$$

Then the two 2 -forms $\omega_{Z}$ and $\omega_{Z}^{\prime}$ obtained by Lie derivation of $\omega_{0}$ with respect to $Z$ and $Z^{\prime}$ are given by

$$
\mathcal{L}_{Z} \omega_{0}=\omega_{Z}=-\Omega_{a b}, \quad \mathcal{L}_{Z^{\prime}} \omega_{0}=\omega_{Z}^{\prime}=\Omega_{a b} .
$$

Proposition 6. The Hamiltonian vector field $\Gamma_{K}$ of the Kepler problem is quasi-Hamiltonian with respect to the complex 2-form $\Omega_{a b}$.

Proof. This can be proved by a direct computation

$$
i\left(\Gamma_{K}\right) \Omega_{a b}=\Gamma_{K}\left(M_{a}\right) d M_{b}^{*}-\Gamma_{K}\left(M_{b}^{*}\right) d M_{a}=\left(i \lambda M_{a}\right) d M_{b}^{*}+\left(i \lambda M_{b}^{*}\right) d M_{a}=i \lambda d\left(M_{a} M_{b}^{*}\right) .
$$

The complex 2-form $\Omega_{a b}$ can be decomposed as

$$
\Omega_{a b}=\Omega_{a b 1}+i \Omega_{a b 2}
$$

where the two real 2-forms, $\Omega_{a b 1}=\operatorname{Re}\left(\Omega_{a b}\right)$ and $\Omega_{a b 2}=\operatorname{Im}\left(\Omega_{a b}\right)$, take the form

$$
\Omega_{a b 1}=d M_{a 1} \wedge d M_{b 1}+d M_{a 2} \wedge d M_{b 2}, \quad \Omega_{a b 2}=-d M_{a 1} \wedge d M_{b 2}+d M_{a 2} \wedge d M_{b 1},
$$


Considering the real and imaginary parts we obtain

$$
i\left(\Gamma_{K}\right) \Omega_{a b 1}=-\lambda d K_{4}, \quad i\left(\Gamma_{K}\right) \Omega_{a b 2}=\lambda d K_{3},
$$

what means that $\Gamma_{K}$ is also quasi-bi-Hamiltonian with respect to the two real 2 -forms $\left(\omega_{0}, \Omega_{a b 1}\right)$ and $\left(\omega_{0}, \Omega_{a b 2}\right)$.

Once more we obtain that the factor $\lambda$ determines that the system is quasi-bi-Hamiltonian instead of just bi-Hamiltonian.

The complex 2 -form $\Omega_{a b}$ is well defined but it is not symplectic. The kernel is two-dimensional and it is invariant under the action of $\Gamma_{K}$

$\left[\operatorname{Ker} \Omega_{a b}, \Gamma_{K}\right] \subset \operatorname{Ker} \Omega_{a b}$.

The coordinate expressions of $\Omega_{a b 1}$ and $\Omega_{a b 2}$ are

$$
\begin{aligned}
& \Omega_{a b 1}=\frac{2 J}{\left(a^{2}+b^{2}\right)^{2}}\left(\alpha_{13} d a \wedge d p_{a}+\alpha_{14} d a \wedge d p_{b}+\alpha_{23} d b \wedge d p_{a}+\alpha_{24} d b \wedge d p_{b}+\alpha_{34} d p_{a} \wedge d p_{b}\right), \\
& \Omega_{a b 2}=\frac{2 g}{\left(a^{2}+b^{2}\right)^{2}}\left(\beta_{13} d a \wedge d p_{a}+\beta_{14} d a \wedge d p_{b}+\beta_{23} d b \wedge d p_{a}+\beta_{24} d b \wedge d p_{b}\right),
\end{aligned}
$$

with $\alpha_{i j}$ and $\beta_{i j}$ being given by

$$
\begin{array}{ll}
\alpha_{13}=\left(2 g b-a p_{a} p_{b}-b p_{b}^{2}\right) b, & \alpha_{14}=-\left(2 g a-a p_{a}^{2}-b p_{a} p_{b}\right) b, \\
\alpha_{23}=\left(-2 g b+a p_{a} p_{b}+b p_{b}^{2}\right) a, & \alpha_{24}=\left(2 g a-a p_{a}^{2}-b p_{a} p_{b}\right) a, \\
\alpha_{34}=2 J\left(a^{2}+b^{2}\right), &
\end{array}
$$

and

$$
\begin{array}{ll}
\beta_{13}=\left(2 a b p_{a}-a^{2} p_{b}-b^{2} p_{b}\right) b, & \beta_{14}=\left(2 a b p_{b}-a^{2} p_{a}-b^{2} p_{a}\right) b, \\
\beta_{23}=\left(-2 a b p_{a}+a^{2} p_{b}+b^{2} p_{b}\right) a, & \beta_{24}=\left(-2 a b p_{b}-a^{2} p_{a}-b^{2} p_{a}\right) a .
\end{array}
$$

We close this section with the following properties:

(i) The two real 2-forms are not symplectic. In fact we have verified that $\Omega_{1} \wedge \Omega_{1}=0$, $\Omega_{2} \wedge \Omega_{2}=0$, and also $\Omega_{1} \wedge \Omega_{2}=0$.

(ii) These two 2-forms, $\Omega_{a b 1}$ and $\Omega_{a b 2}$, determine two recursion operators $((1,1)$ tensor fields) $R_{a b 1}$ and $R_{a b 2}$ defined as

$$
\Omega_{a b 1}(X, Y)=\omega_{0}\left(R_{a b 1} X, Y\right), \quad \Omega_{a b 2}(X, Y)=\omega_{0}\left(R_{a b 2} X, Y\right),
$$

or in an equivalent way

$$
R_{a b 1}={\widehat{\omega_{0}}}^{-1} \circ \widehat{\Omega_{a b 1}}, \quad R_{a b 2}={\widehat{\omega_{0}}}^{-1} \circ \widehat{\Omega_{a b 2}} .
$$

As in Section 2.3, a consequence of the singular character of $\Omega_{a b 1}$ and $\Omega_{a b 2}$ is that

$$
\operatorname{det}\left[R_{a b 1}\right]=\operatorname{det}\left[R_{a b 2}\right]=0 .
$$

(iii) If we denote by $Z_{3}$ and $Z_{4}$ the Hamiltonian vector fields (with respect to the canonical symplectic form $\omega_{0}$ ) of the integrals $K_{3}$ and $K_{4}$, then the dynamical vector field $\Gamma_{K}$ is orthogonal to $Z_{4}$ with respect to the structure $\Omega_{1}$ and it is also orthogonal to $Z_{3}$ with respect to the structure $\Omega_{2}$, that is,

$$
i\left(\Gamma_{K}\right) i\left(Y_{4}\right) \Omega_{a b 1}=0, \quad i\left(\Gamma_{K}\right) i\left(Y_{3}\right) \Omega_{a b 2}=0 .
$$




\section{Final comments}

The Kepler problem is separable in two different coordinate systems, and because of this, it is superintegrable with quadratic integrals of motion. Now we have proved that this superintegrability is directly related with the existence of certain complex functions possessing very interesting Poisson bracket properties and also that these functions are also related with the existence of quasi-bi-Hamiltonian structures.

We finalize with some questions for future work. First, as stated in the Introduction, quasibi-Hamiltonian structures is a matter that still remain as slightly studied (in contrast to the bi-Hamiltonian systems); so the particular case of the Kepler problem can be a good motivation to undertake a better study of these systems. Second, the existence of these complex functions is not a specific characteristic of the Kepler problem; in fact, it has been proved that the superintegrability of certain systems recently studied (as the isotonic oscillator or the TTW or PW systems) [11, 29, 30, 31, 32, 33] is also related with such a class of complex functions. Therefore, an interesting open question is whether these other superintegrable systems are also endowed with bi-Hamiltonian structures or with quasi-bi-Hamiltonian structures; the starting point for this study must be a deeper analysis of the properties of these complex functions making use of the geometric (symplectic) formalism as an approach.

\section{Acknowledgments}

This work has been supported by the research projects MTM-2012-33575 (MICINN, Madrid) and DGA-E24/1 (DGA, Zaragoza).

\section{References}

[1] Błaszak M., Bi-Hamiltonian representation of Stäckel systems, Phys. Rev. E 79 (2009), 056607, 9 pages, arXiv:0904.2070.

[2] Boualem H., Brouzet R., Rakotondralambo J., Quasi-bi-Hamiltonian systems: why the Pfaffian case?, Phys. Lett. A 359 (2006), 559-563.

[3] Boualem H., Brouzet R., Rakotondralambo J., About the separability of completely integrable quasi-biHamiltonian systems with compact levels, Differential Geom. Appl. 26 (2008), 583-591.

[4] Brouzet R., Caboz R., Rabenivo J., Ravoson V., Two degrees of freedom quasi-bi-Hamiltonian systems, J. Phys. A: Math. Gen. 29 (1996), 2069-2076.

[5] Cariñena J.F., Guha P., Rañada M.F., Quasi-Hamiltonian structure and Hojman construction, J. Math. Anal. Appl. 332 (2007), 975-988.

[6] Cariñena J.F., Guha P., Rañada M.F., Hamiltonian and quasi-Hamiltonian systems, Nambu-Poisson structures and symmetries, J. Phys. A: Math. Theor. 41 (2008), 335209, 11 pages.

[7] Cariñena J.F., Ibort L.A., Non-Noether constants of motion, J. Phys. A: Math. Gen. 16 (1983), 1-7.

[8] Cariñena J.F., Marmo G., Rañada M.F., Non-symplectic symmetries and bi-Hamiltonian structures of the rational harmonic oscillator, J. Phys. A: Math. Gen. 35 (2002), L679-L686, hep-th/0210260.

[9] Cariñena J.F., Rañada M.F., Canonoid transformations from a geometric perspective, J. Math. Phys. 29 (1988), 2181-2186.

[10] Cariñena J.F., Rañada M.F., Generating functions, bi-Hamiltonian systems, and the quadratic-Hamiltonian theorem, J. Math. Phys. 31 (1990), 801-807.

[11] Cariñena J.F., Rañada M.F., Santander M., Two important examples of nonlinear oscillators, in Proceedings of 10th International Conference in Modern Group Analysis (Larnaca, Cyprus, 2004), Editors N.H. Ibragimov, C. Sophocleous, P.A. Damianou, 2005, 39-46, math-ph/0505028.

[12] Crampin M., Marmo G., Rubano C., Conditions for the complete integrability of a dynamical system admitting alternative Lagrangians, Phys. Lett. A 97 (1983), 88-90.

[13] Crampin M., Prince G.E., Alternative Lagrangians for spherically symmetric potentials, J. Math. Phys. 29 (1988), 1551-1554. 
[14] Crampin M., Sarlet W., A class of nonconservative Lagrangian systems on Riemannian manifolds, J. Math. Phys. 42 (2001), 4313-4326.

[15] Crampin M., Sarlet W., Bi-quasi-Hamiltonian systems, J. Math. Phys. 43 (2002), 2505-2517.

[16] Crampin M., Sarlet W., Thompson G., Bi-differential calculi and bi-Hamiltonian systems, J. Phys. A: Math. Gen. 33 (2000), L177-L180.

[17] De Filippo S., Vilasi G., Marmo G., Salerno M., A new characterization of completely integrable systems, Nuovo Cimento B 83 (1984), 97-112.

[18] Fernandes R.L., Completely integrable bi-Hamiltonian systems, J. Dynam. Differential Equations 6 (1994), 53-69.

[19] Grigoryev Y.A., Tsiganov A.V., On bi-Hamiltonian formulation of the perturbed Kepler problem, J. Phys. A: Math. Theor. 48 (2015), 175206, 7 pages, arXiv:1504.02211.

[20] Henneaux M., Shepley L.C., Lagrangians for spherically symmetric potentials, J. Math. Phys. 23 (1982), 2101-2107.

[21] Marmo G., A geometrical characterization of completely integrable systems, in Proceedings of the International Meeting on Geometry and Physics (Florence, 1982), Pitagora, Bologna, 1983, 257-262.

[22] McLenaghan R.G., Smirnov R.G., A class of Liouville-integrable Hamiltonian systems with two degrees of freedom, J. Math. Phys. 41 (2000), 6879-6889.

[23] Morosi C., Tondo G., Quasi-bi-Hamiltonian systems and separability, J. Phys. A: Math. Gen. 30 (1997), 2799-2806, solv-int/9702006.

[24] Morosi C., Tondo G., On a class of dynamical systems both quasi-bi-Hamiltonian and bi-Hamiltonian, Phys. Lett. A 247 (1998), 59-64, solv-int/9811007.

[25] Rakotondralambo J., A cohomological obstruction for global quasi-bi-Hamiltonian fields, Phys. Lett. A 375 (2011), 1064-1068.

[26] Rañada M.F., Alternative Lagrangians for central potentials, J. Math. Phys. 32 (1991), 2764-2769.

[27] Rañada M.F., Dynamical symmetries, bi-Hamiltonian structures, and superintegrable $n=2$ systems, J. Math. Phys. 41 (2000), 2121-2134.

[28] Rañada M.F., A system of $n=3$ coupled oscillators with magnetic terms: symmetries and integrals of motion, SIGMA 1 (2005), 004, 7 pages, nlin.SI/0511007.

[29] Rañada M.F., A new approach to the higher order superintegrability of the Tremblay-Turbiner-Winternitz system, J. Phys. A: Math. Theor. 45 (2012), 465203, 9 pages, arXiv:1211.2919.

[30] Rañada M.F., Higher order superintegrability of separable potentials with a new approach to the PostWinternitz system, J. Phys. A: Math. Theor. 46 (2013), 125206, 9 pages, arXiv:1303.4877.

[31] Rañada M.F., The Tremblay-Turbiner-Winternitz system on spherical and hyperbolic spaces: superintegrability, curvature-dependent formalism and complex factorization, J. Phys. A: Math. Theor. 47 (2014), 165203, 9 pages, arXiv:1403.6266.

[32] Rañada M.F., The Post-Winternitz system on spherical and hyperbolic spaces: a proof of the superintegrability making use of complex functions and a curvature-dependent formalism, Phys. Lett. A 379 (2015), $2267-2271$.

[33] Rañada M.F., Rodríguez M.A., Santander M., A new proof of the higher-order superintegrability of a noncentral oscillator with inversely quadratic nonlinearities, J. Math. Phys. 51 (2010), 042901, 11 pages, arXiv:1002.3870.

[34] Rauch-Wojciechowski S., A bi-Hamiltonian formulation for separable potentials and its application to the Kepler problem and the Euler problem of two centers of gravitation, Phys. Lett. A 160 (1991), 149-154.

[35] Tempesta P., Tondo G., Generalized Lenard chains, separation of variables, and superintegrability, Phys. Rev. E 85 (2012), 046602, 11 pages, arXiv:1205.6937.

[36] Zeng Y.B., Ma W.X., Families of quasi-bi-Hamiltonian systems and separability, J. Math. Phys. 40 (1999), 4452-4473, solv-int/9906005. 